\title{
Carbon Nanotubes Interconnects for Nanoelectronics Circuits
}

\author{
A.G. Chiariello*, A. Maffucci ${ }^{\circ}$, G. Miano* and F. Villone ${ }^{\circ}$ \\ *DIEL, Università di Napoli "Federico II", Napoli \\ ${ }^{\circ}$ DAEIMI, Università di Cassino, Cassino \\ Italy
}

\section{Introduction}

Future nanoelectronics will be enabled only by providing the effective capability of connecting the nanometric devices to the circuit boards, therefore a major challenge is the design and fabrication of the nano-interconnects. For nanotechnology applications the limits imposed by physics, materials, assembly and design could not be overcome by simply scaling the conventional metal/dielectric systems: innovation in new materials, new technology and new system integration techniques is required.

Carbon nanotubes (CNTs) are recently discovered carbon structures (Iijima, 1991), which have been soon considered as emerging research materials (ITRS, 2007) for nanoelectronics applications (Fig.1), because of their unique properties (Avouris et al., 2003; Saito et al., 2004; Anantram \& Léonard, 2006). CNT interconnects are expected to meet many of the requirements for technologies below the 22nm node (ITRS, 2007) in terms of mechanical strength, thermal conductivity and electrical performances.

This Chapter is devoted to CNT interconnects, proposed for wiring and for packaging nanotechnology ICs, with the aim of presenting the state-of-the-art of electrodynamics and circuit modelling of CNTs and to provide performance comparisons between conventional and CNT interconnects. Some case-studies of practical application are carried-out, referring to real-world wiring and packaging problems for future nanoelectronics.

(a)

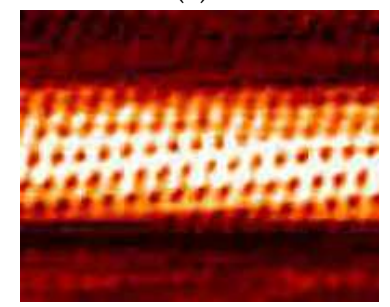

(b)

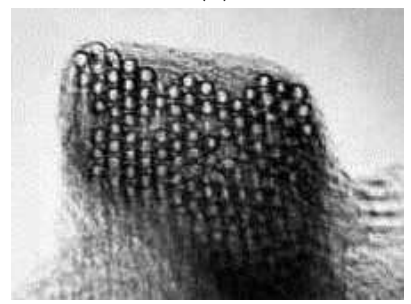

(c)

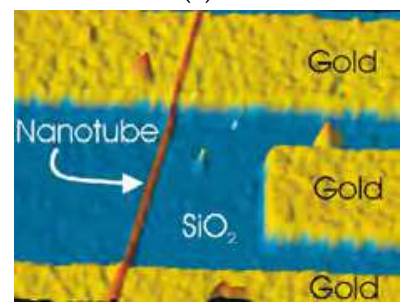

Fig. 1. Some real-world nanotubes: (a) AFM image of chiral tube of $1.3 \mathrm{~nm}$ diameter (Technical University, Delft); (b) TEM image of a crystalline nanotube bundle (Rice University); (c) a single nanotube as interconnect between gold electrodes (IBM). 
Due to the potential applications of CNTs in nanoelectronics, many efforts have been made in literature to derive models able to describe the electrical propagation. Examples of phenomenological models are found in (Burke, 2002; Burke, 2003), based on the Luttinger liquid theory. Semi-classical approaches describing the CNTs as electron waveguides may be found in (Wesström, 1996; Sarto, 2009), and in (Salahuddin, 2005), where a general model for a quantum wire is derived from the transport theory based on the Boltzmann equation. In this Chapter we adopt the semi-classical model first proposed in (Miano \& Villone, 2006), based on a fluid description of the motion of the CNT free electrons. This model has been used to derive transmission line equivalent circuits for metallic CNT interconnects in (Maffucci et al, 2008a; Maffucci et al., 2009), and has been recently extended to CNTs of arbitrary chirality (Forestiere et al., 2009a and 2009b). Note that the same model may be extended to take into account also the effect of CNT curvature (Forestiere et al., 2009b)

Using any of the quoted models, the comparative analysis between CNT and conventional $\mathrm{Cu}$ interconnects leads to a quite general result: nano-interconnects made by good quality $\mathrm{CNT}$ bundles outperform $\mathrm{Cu}$ ones at intermediate and global level, whereas at local level the behaviour is comparable (Li et al., 2009; Maffucci et al., 2008b; Naeemi, 2008). Therefore, the focus is now on the availability of a low-cost fabrication process providing CNT bundles with low contact resistance, good densities, good direction control and compatibility with CMOS technology. Satisfactorily results have been achieved for vertical CNT bundles (ITRS, 2007; Li et al., 2009; Liu, 2009). The same good results, however, have not yet been obtained in the fabrication of $\mathrm{CNT}$ interconnects parallel to the substrate, which remains a challenging task. On the other hand, many of the problems related to the integration between the CNT technology and the CMOS one are still open. However, the gap between theoretical predictions and practical applications is reducing faster and faster, and many examples have been recently given of the first real-world applications of CNT interconnects. In the nanopackaging area CNT bumps for flip-chip interconnects have been fabricated, replacing the conventional solder bumps, Fig.2a (Soga, 2008). Recently, a $1.02 \mathrm{GHz}$ oscillator ring circuit using CNT wiring for a conventional chip has been presented, see Fig.2b (Close et al., 2008). These are only few of the many examples of successful integration between CNTs and ICs, testimonials of this technological trend.

(a)

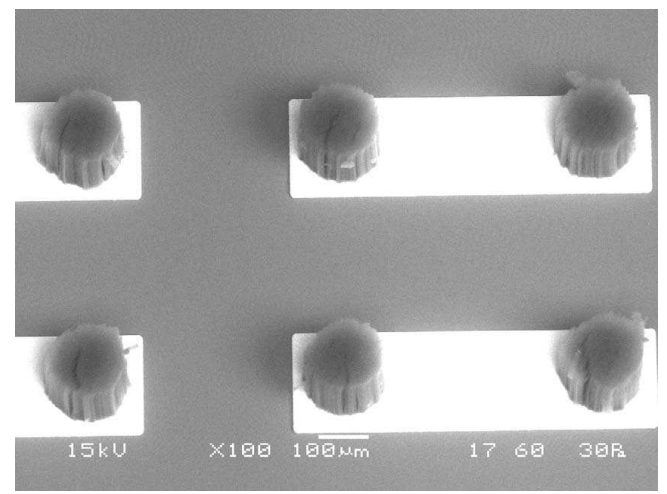

(b)

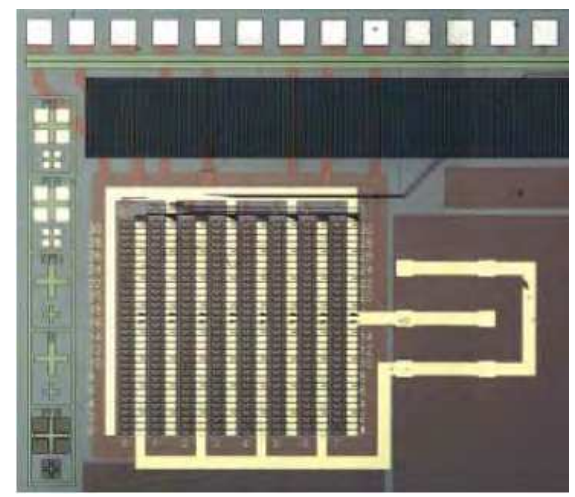

Fig. 2. (a) Vertical CNT bundles as flip-chip bumps (Fujitsu Labs.); (b) carbon nanotubes wiring in an oscillator circuit (Stanford University $\mathcal{E}$ Toshiba). 


\section{Electromagnetic propagation models for CNTs}

A carbon nanotube is realized by rolling-up a sheet of a mono-atomic layer of graphite (graphene), see Fig.3a. The rolled graphene may become either metallic or semiconducting, depending on its chirality, i.e. the way in which it is rolled up. A single-wall carbon nanotube (SWCNT) is made by a single sheet of graphene (Fig.3b), whereas a multi-wall carbon nanotube (MWCNT) is made by nested sheets (Fig.3c). In the graphene the conduction phenomenon is related to the motion of the so-called $\pi$-electron, i.e. the one out of the four valence electrons which is free to move between the positive ion lattice. Both semiclassical as well as quantum-mechanical approaches have been used to describe the CNT electron dynamics.

We study the electrodynamics of a CNT shell, which may either describe an isolated SWCNT or a shell of a MWCNT, in which the direct coupling of electronic states of adjacent shells may be disregarded. The electrical signal propagation along the CNT shell is analyzed assuming a low frequency regime, where only intraband transitions of $\pi$-electrons with unchanged transverse quasimomentum occur. This allows using the semi-classical transport theory expounded, for instance, in (Slepyan et al., 1999; Maksimenko et al., 2000; Miano \& Villone, 2006: Maffucci et al., 2008a; Forestiere et al., 2009a and 2009b). Using this approach, the longitudinal dynamic conductivity of a CNT shell of arbitrary chirality may be obtained, expressed in terms of the number of effective conducting channels. This leads to the constitutive equation of this innovative material, which could be coupled to Maxwell equations in order to derive electromagnetic and/or circuital models, following the stream of what done in (Miano \& Villone, 2006: Maffucci et al., 2008a; Forestiere et al., 2009).

\subsection{Constitutive relation of a CNT shell}

The chirality of a CNT is described by two integers $n$ and $m$, which are the coefficients of he basis vectors $\mathbf{a}_{1}$ and $\mathbf{a}_{2}$ depicted in Fig.3a. The CNT is metallic if $|n-m|=3 q$, where $q=0,1,2 \ldots$ A zig-zag CNT is obtained for $n=0$ or $m=0$, whereas an armchair CNT is given by $n=m$, therefore, armchair CNTs are always metallic, whereas zig-zag ones are metallic only if $m=3 q$, with $q=0,1,2 \ldots$ For $m \rightarrow \infty$ or $n \rightarrow \infty$ any CNT tends to the graphene sheet. The CNT diameter $D$ is given by:

$$
D=0.0782 \sqrt{n^{2}+n m+m^{2}} \quad[\mathrm{~nm}] .
$$

(a)

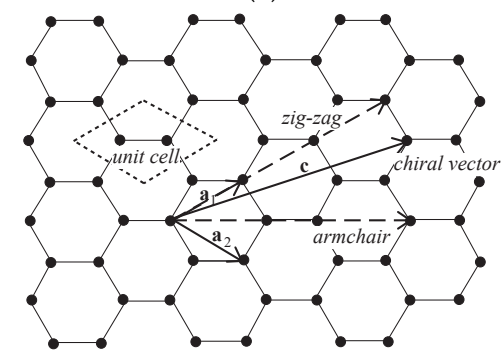

(b)

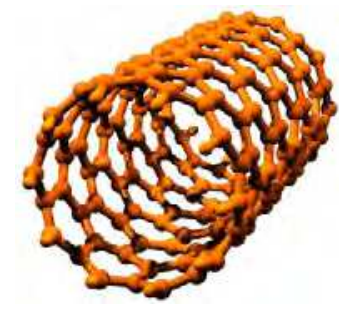

(c)

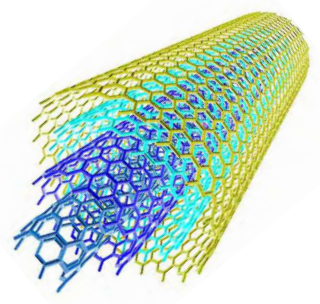

Fig. 3. (a) the graphene lattice; (b) single-wall CNT; (c) multi-wall CNT. 
For each segment of the first Brillouin zone of the nanotube we introduce a distribution function for the $\pi$ electrons in the valence and conduction bands:

$$
f_{\mu}^{( \pm)}=f_{\mu}^{( \pm)}(z, k, t), \quad \mu=0,1, \ldots N,-\frac{\pi}{T}<k \leq \frac{\pi}{T}
$$

where $k$ is the longitudinal wavenumber, $t$ is the time, $N$ is the number of graphene exagons in the primitive cell of the CNT, and $T$ is the translational length. By assuming that the electric field is transversally uniform, $f_{\mu}^{( \pm)}$satisfy the quasi-classic Boltzmann equation

$$
\frac{\partial f_{\mu}^{( \pm)}}{\partial t}+v_{\mu}^{( \pm)} \frac{\partial f_{\mu}^{( \pm)}}{\partial z}+\frac{2 \pi e}{h} E_{z} \frac{\partial f_{\mu}^{( \pm)}}{\partial k}=-v\left(f_{\mu}^{( \pm)}-f_{0, \mu}^{( \pm)}\right)
$$

where $e$ is the electron charge, $\mathrm{h}$ is the Planck constant, $E_{z}(z, t)$ is the component of the electric field tangent to the CNT surface, $v$ is the frequency of collision of the electrons with the ion lattice, $v_{\mu}^{( \pm)}$are the longitudinal velocities of the electrons in the conduction/valence subbands and $f_{0, \mu}^{( \pm)}$are the distribution functions at equilibrium. The latter are given by:

$$
f_{0, \mu}^{( \pm)}(k)=\frac{1}{\pi^{2} D} F\left[E_{\mu}^{( \pm)}(k)\right], \quad F[E]=\frac{1}{e^{E / k_{B} T_{0}}+1}
$$

being $E_{\mu}^{( \pm)}$the energy of the generic subband, $F[E]$ the Dirac-Fermi function, $k_{B}$ the Boltzmann constant and $T_{0}$ the $\mathrm{CNT}$ absolute temperature. Let us assume time-harmonic electric field and surface current density:

$$
E_{z}(z, t)=\operatorname{Re}\left\{\hat{E}_{z} e^{i(\omega t-\beta z)}\right\}, \quad J_{z}(z, t)=\operatorname{Re}\left\{\hat{J}_{z} e^{i(\omega t-\beta z)}\right\} .
$$

For small perturbations of functions (2) around the equilibrium values (4) we get

$$
\hat{E}_{z}=\hat{\sigma}_{z z}(\beta, \omega) \hat{J}_{z}
$$

where the spectral domain conductivity $\hat{\sigma}_{z z}(\beta, \omega)$ is given by

$$
\hat{\sigma}_{z z}(\beta, \omega)=\frac{2 i e^{2}}{\pi^{2} D} \sum_{\mu=0}^{N-1} \int_{0}^{\pi / T}\left(\frac{1}{\omega-v_{\mu}^{(+)} \beta-i v}+\frac{1}{\omega+v_{\mu}^{(+)} \beta-i v}\right) v_{\mu}^{(+) 2} \frac{d F}{d E_{\mu}^{(+)}} d k
$$

For all the subbands that give a meaningful contribution to the conductivity we may assume $v_{\mu}^{( \pm)} \cong v_{F}$ in (7), being $v_{F}$ the Fermi velocity. This leads to

$$
\hat{\sigma}_{z z}(\beta, \omega)=\frac{4 e^{2} v_{F}}{\pi h D} \frac{1}{i \omega+v} \frac{1}{1+v_{F}^{2} \beta^{2} /(i \omega+v)^{2}} M(n, m),
$$


where $M$ may be regarded as the equivalent number of conducting channels:

$$
M(n, m)=\frac{h}{\pi v_{F}} \sum_{\mu=0}^{N-1} \int_{0}^{\pi / T} v_{F}^{2}\left(\frac{d F}{d E_{\mu}^{+}}\right) d k .
$$

The constitutive relation of the CNT in the frequency domain comes from (6), (8) and (9):

$$
(1+i \omega / v) J_{z}+\frac{v_{F}^{2}}{v(1+v / i \omega)} \frac{\partial \rho_{s}}{\partial z}=\sigma_{c} E_{z}
$$

where $\rho_{s}$ is the surface charge density and $\sigma_{c}$ is the static limit for the axial conductivity:

$$
\sigma_{c}=\frac{4 e^{2} v_{F}}{v \pi h D} M(n, m)
$$

In the limit $\omega / v>>1$, (11) reduces to the simple fluid model in (Miano \& Villone, 2006; Maffucci et al., 2008a) and for $\omega / v<<1$ the electrons behave as a cold fluid.

\subsection{Approximation of the equivalent number of conducting channels}

The number of conducting channels $M$ is a function of the parameters affecting the number of subbands around the CNT Fermi level: CNT chirality, diameter and temperature. Figure 4a shows $M$ for increasing $C N T$ diameter, evaluated at $T=273 \mathrm{~K}$. For small diameters it is $M=2$ for metallic CNTs and $M=0$ for semiconducting ones, i.e. only metallic CNTs contribute the conduction. For larger diameters the channels of a metallic CNT increase and those associated to semiconducting ones are no longer negligible.

Let us now compute the axial conductivity for the two cases: Fig.4b shows these values, normalized to the axial conductivity for a graphene sheet, given by (Slepyan, 1999):

$$
\sigma_{\infty}=\frac{8 \pi \ln 2}{h^{2}} \frac{e^{2} k_{B} T}{v+i \omega} .
$$

Figure $4 \mathrm{~b}$ agrees with the condition that for $D \rightarrow \infty$ a CNT tends to the graphene.

To obtain a simple analysis tool for studying MWCNTs or bundles of CNTs, it is useful to fit the behavior of $M$ through a simple approximated function of the diameter $D$ and temperature T. A piecewise linear function able to fit the asymptotic behaviors for $D \rightarrow \infty$ and $D \rightarrow 0$ may be given by the following expression (the parameters are given in Table 1 ):

$$
M \approx\left\{\begin{array}{ll}
M_{0} & \text { for } D<d_{0} / T \\
a_{1} D T+a_{2} & \text { for } D \geq d_{0} / T
\end{array} .\right.
$$

Figure 5 shows the fitting results computed at $T=273 \mathrm{~K}$ and $T=373 \mathrm{~K}$.

This result is consistent with similar approximations proposed in literature (Naeemi, 2008), where however $M$ is sligthly overestimated, due to the approximations used in evaluating the number of subbands around the Fermi level. 


\begin{tabular}{|l|c|c|}
\hline & Metallic & Semiconducting \\
\hline$M_{0}$ [adim.] & 2 & 0 \\
\hline$a_{1}[1 / \mathrm{nmK}]$ & $3.3 \cdot 10^{-4}$ & $3.3 \cdot 10^{-4}$ \\
\hline$a_{2}$ [adim.] & 0.15 & -0.20 \\
\hline$d_{0}$ [nm.K] & $5.6 \cdot 10^{3}$ & $0.6 \cdot 10^{3}$ \\
\hline
\end{tabular}

Table 1. Parameters for the fitting formulas (13).

(a)

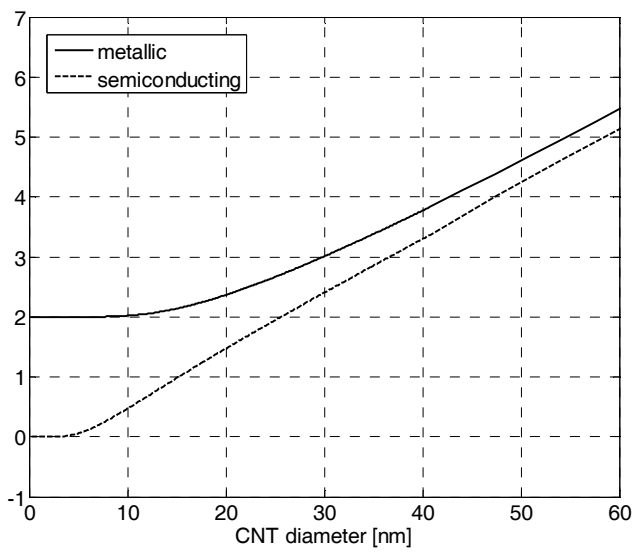

(b)

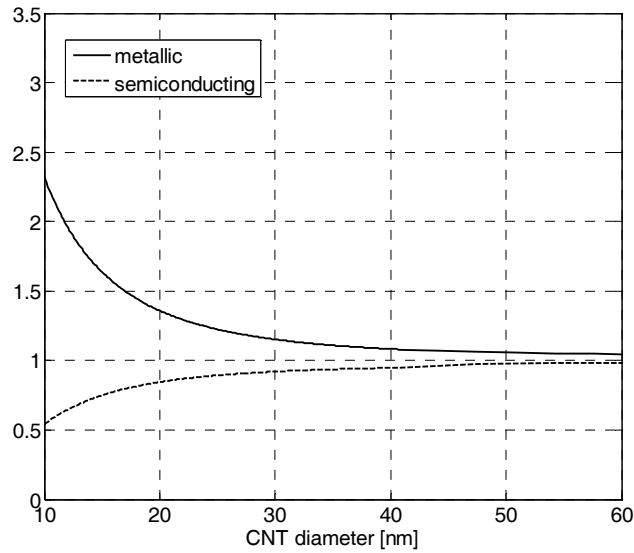

Fig. 4. (a) Number of effective conducting channels versus CNT diameter, at $T=273 \mathrm{~K}$; (b) axial conductivity normalized to the graphene value.

(a)

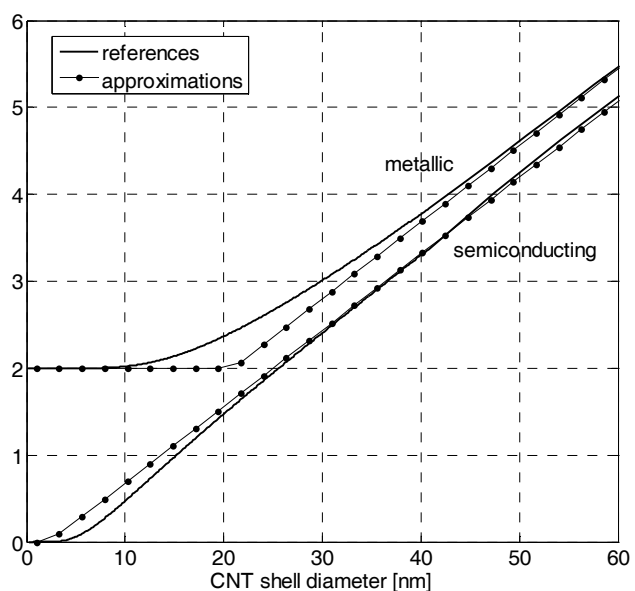

(b)

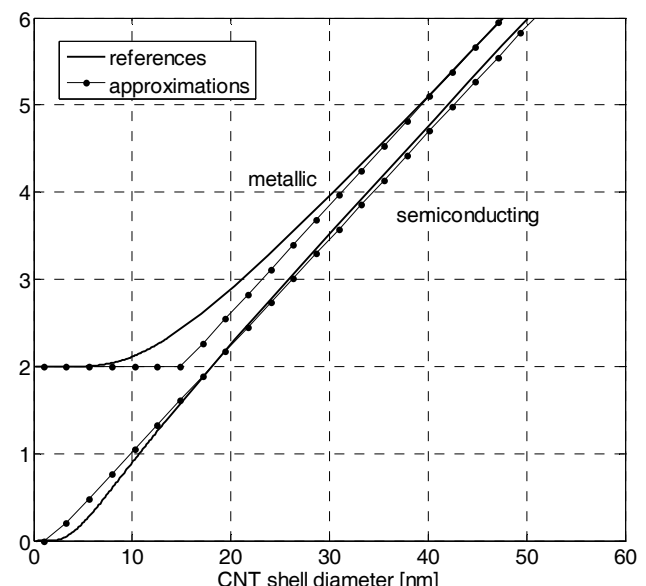

Fig. 5. Piecewise linear fitting (13) for the number of effective conducting channels evaluated at: (a) $T=273 \mathrm{~K}$; and (b) $T=373 \mathrm{~K}$. 


\section{Circuit models for CNT bundle interconnects}

\subsection{Circuit model for a single CNT above a ground plane}

A circuit model for CNT interconnects may be derived in the framework of the transmission line (TL) theory. The problem may be formulated in frequency domain by coupling Maxwell equations to the CNT constitutive relation (10). Then, assuming the propagation to be of quasi-TEM type, a distributed RLC circuit may be derived.

Let us first consider the simple case of a CNT shell of diameter $D$, located at a distance $t$ above a PEC ground, Fig. 6a. As shown in paragraph 4.3, the distribution of surface currents and charges along the contour of a CNT section may be assumed to be uniform, since the proximity effect is negligible, hence the current and charge at any position along the CNT axis are given by:

$$
Q(\omega, z)=\pi \mathrm{D} \rho_{s}(\omega, z), \quad I(\omega, z)=\pi \mathrm{D} J_{z}(\omega, z) .
$$

Using (14) in (10) we get:

$$
(v+i \omega) I(\omega, z)+\frac{v_{F}^{2}}{(1+v / i \omega)} \frac{\partial Q(\omega, z)}{\partial z}=2 v_{F} \frac{M}{R_{0}} E_{z}(\omega, z),
$$

being $R_{0}=h / 2 e^{2}=12.9 \mathrm{k} \Omega$ the so-called quantum resistance. The longitudinal component of the electric field $E_{z}$ may be derived from the scalar electric and vector magnetic potentials:

$$
E_{z}(\omega, z)=-\frac{\partial V(\omega, z)}{\partial z}-i \omega A_{z}
$$

which may be related to charge and current through the classical expressions:

$$
V(\omega, z)=\frac{Q(\omega, z)}{C_{e}}, \quad A_{z}(\omega, z)=L_{m} I(\omega, z),
$$

being $C_{e}, L_{m}$ the per-unit-length (pul) electrostatic capacitance and magnetic inductance.

(a)

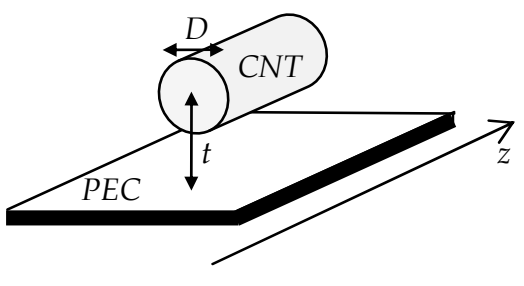

(b)

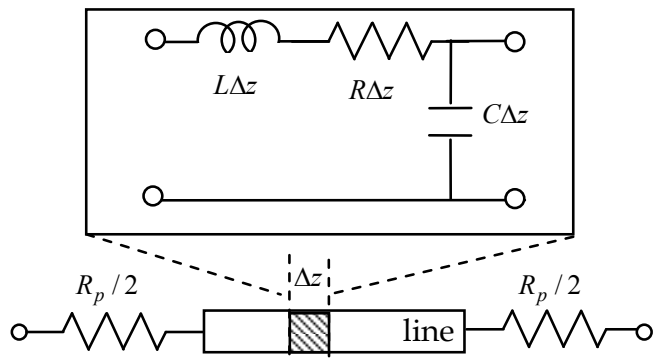

Fig. 6. (a) A CNT shell above a PEC; (b) equivalent circuit: elementary cell (inset) and lumped contact resistances. 
Combining (15)-(17) we get the first Telegraphers' equation:

$$
-\frac{d V(\omega, z)}{d z}=(R(\omega)+i \omega L(\omega)) I(\omega, z),
$$

with the pul parameters $R$ and $L$ defined as:

$$
L=\left(L_{m}+L_{k}\right) / a_{C}, \quad R=v L_{k} / a_{C}, \quad C=C_{e},
$$

where the parameter $\alpha_{C}$ is given by:

$$
a_{C}=1+\frac{C_{e}}{C_{q}} \frac{1}{1+v / i \omega}
$$

In (19) we have introduced the p.u.l. kinetic inductance $L_{k}$ and p.u.l. quantum capacitance $C_{q}$ :

$$
L_{k}=\frac{R_{0}}{2 v_{F} M}=\frac{h}{4 e^{2} v_{F} M}, \quad C_{q}=\frac{1}{L_{k} v_{F}^{2}}=\frac{2 M}{R_{0} v_{F}}=\frac{4 e^{2} M}{h v_{F}}
$$

The second Telegraphers' equation is obtained by imposing the charge conservation law:

$$
-\frac{d I(\omega, z)}{d z}=i \omega C V(\omega, z)
$$

with the pul capacitance $C$ defined as $C=C_{e}$.

Equations (18) and (22) describe a lossy TL where the quantum effects are combined to the classical electrical and magnetic ones in the definition of the p.u.l. parameters $R, L$, and $C$. Assuming a frequency operating condition such as $v / \omega<<1$, the parameters $R$ and $L$ do not depend on frequency, since (20) becomes:

$$
a_{C} \approx 1+C_{e} / C_{q} .
$$

Typical orders of magnitude for the collision frequency $v$ are $10^{11} \div 10^{12} \mathrm{~Hz}$, hence the above condition is satisfied for frequencies up to the order of hundrends of GHz. Assuming this limit, the propagation along a CNT is then described by a simple lossy TL with constant parameters $R, L$, and $C$. The elementary cell is depicted in the inset of Fig.6b. The circuit model obtained here is a generalization of other models proposed in literature: for instance in (Burke, 2003; Salahuddin, 2005; Maffucci et al., 2008a) TL models for metallic SWCNTs are obtained. The elementary cell is however slightly different from those used in (Burke, 2003; Salahuddin, 2005), where the voltage variable is derived from the electrochemical potential rather than from the electrostatic one. A detailed discussion may be found in (Maffucci et al., 2009). The TL models in the quoted references describe metallic CNTs, where the model presented here is more general, since it deals with both metallic and semiconducting CNTs of any diameter. For instance the values of $L_{k}$ and $C_{q}$ used in literature may be obtained using $M=2$ in (21), i.e. assuming metallic CNT with small radius: 


$$
L_{k 0}=\frac{h}{8 e^{2} v_{F}}=\frac{R_{0}}{4 v_{F}}, \quad C_{q 0}=\frac{8 e^{2}}{h v_{F}}=\frac{4}{R_{0} v_{F}}
$$

Assuming in (24) $c_{s} \approx v_{F} \approx 8 \cdot 10^{5} \mathrm{~m} / \mathrm{s}$, we get $L_{k 0} \approx 4 \mathrm{nH} / \mu \mathrm{m}$ and $C_{q 0} \approx 400 \mathrm{aF} / \mu \mathrm{m}$. However the actual values deviate from this bulk values as an effect of the increase of the number of channels with the CNT diameter, as shown in Figure 7.

Let us now investigate the influence of the quantistic capacitance and kinetic inductance on the pul parameters (19). Assuming a homogeneous dielectric the classical parameters are:

$$
L_{m}=\frac{\mu}{2 \pi} \cosh ^{-1}\left(\frac{2 t}{D}\right), \quad C_{e}=2 \pi \varepsilon / \cosh ^{-1}\left(\frac{2 t}{D}\right) .
$$

Let us consider the case of a metallic SWCNT with $D=4 \mathrm{~nm}, \varepsilon_{r}=2.2$ and $\mu=\mu_{0}$. For such a condition $L_{k} \approx L_{k 0}$ and $C_{q} \approx C_{q 0}$. Table 2 shows the ratio between classical and quantistic parameters for different aspect ratios $t / D$. The kinetic inductance dominates the magnetic one, being 3-4 order of

(a)

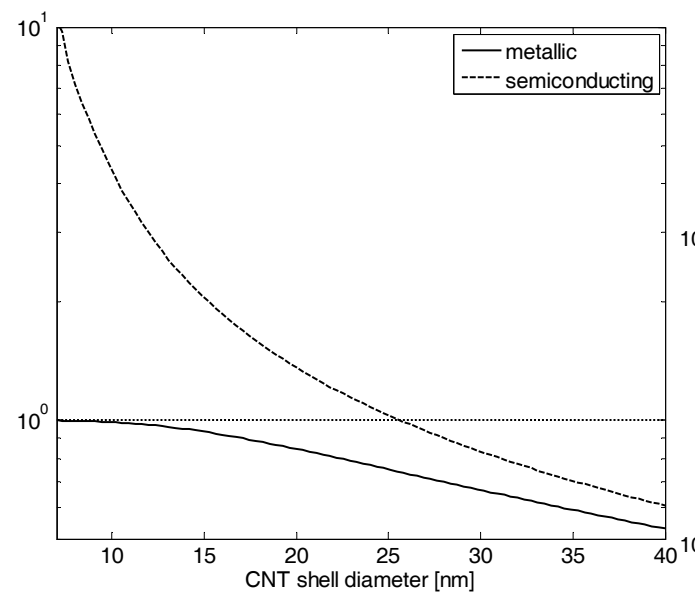

(b)

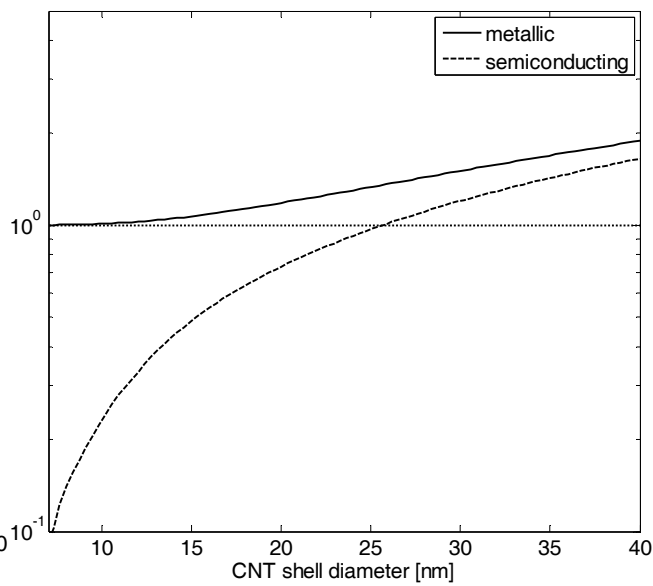

Fig. 7. (a) kinetic inductance, and (b) quantum capacitance for a CNT shell vs diameter, normalized to the bulk values (24).

\begin{tabular}{|c|c|c|c|}
\hline$t / D$ & $L_{k} / L_{\mathrm{m}}$ & $C_{\mathrm{e}} / C_{q}$ & $\alpha_{\mathrm{C}}$ \\
\hline 1.5 & $8.1 \cdot 10^{3}$ & 0.13 & 1.13 \\
\hline 3 & $7.5 \cdot 10^{3}$ & 0.14 & 1.14 \\
\hline 5 & $5.5 \cdot 10^{3}$ & 0.09 & 1.09 \\
\hline 10 & $4.6 \cdot 10^{3}$ & 0.07 & 1.07 \\
\hline
\end{tabular}

Table 2. Comparison between classical and quantistic pul TL parameters for a SWCNT. 
magnitude higher. As for the capacitance, the quantum capacitance is approximately one order of magnitude smaller than the electrostatic one, hence the parameter $\alpha_{C}$ is close to 1 . A consequence of the huge value of the kinetic inductance is a low propagation velocity and an high characteristic impedance: the values of these two parameters normalized to an ideally scaled PEC interconnect of same dimensions would be

$$
\frac{c_{C N T}}{c} \approx \sqrt{\frac{L_{m}}{L_{k}}}, \quad \frac{Z_{C N T}}{Z_{C}} \approx \sqrt{\frac{L_{k}}{L_{m}}} .
$$

Indeed the lower propagation velocity is not a real limit for the practical application of CNT interconnects. Assuming the same conditions as those used for Table 2, for $t=3 D$ it is $c_{C N T} \approx 2.5 \cdot 10^{6} \mathrm{~m} / \mathrm{s}$, instead of $c \approx 2 \cdot 10^{8} \mathrm{~m} / \mathrm{s}$, hence at $f=10 \mathrm{GHz}$ we have a wavelength $\lambda=0.25 \mathrm{~mm}$. This means that at local and semilocal level the line is electrically short and the propagation delay, although higher than that introduced by an ideally scaled conventional interconnect, is negligible compared to the delay introduced by the $R C$ or $R L C$ behavior of the line (e.g., Banerjee, 2008; Maffucci et al., 2008b).

Let us now analyze the p.u.l. resistance, using (19) with $\alpha_{C} \approx 1$ and assumimg $L_{K} \approx L_{k 0}$ :

$$
R=v L_{K}=\frac{R_{0}}{2 M} \frac{1}{l_{m f p}},
$$

where the collision frequency $v$ has been defined as

$$
v=v_{F} / l_{m f p},
$$

being $l_{m f p}$ the mean-free-path. The values of $l_{m f p}$ may vary significantly if low or high bias conditions are considered. In the low bias condition $l_{m f p}$ may be assumed constant, whereas for higher bias conditions, $l_{m f p}$ depends on the longitudinal voltage too, hence the parameter $R$ is a non-linear function of the longitudinal voltage, usually described as a piecewise linear function with only two asymptotic values (Raychowdhury \& Roy, 2006). A detailed discussion on this point may be found in (Park et al., 2004; Maffucci et al., 2009). In addition, assuming a CNT length greater than the ballistic length, a simple linear dependence of $l_{m f p}$ on the diameter $D$ may be considered (Nieuwoudt \& Massoud, (2006).

As a final remark we note that experimental evidences and theoretical limits show a bulk value of $R_{0} / 2 \approx 6.45 \mathrm{k} \Omega$ for the CNT resistance, which is a lower bound even when ballistic transport is considered (e.g., Park et al., 2004). Furthermore, if the contacts between the CNT and the metallic electrodes at its terminations are not ideal, we have to consider an additional parasitic lumped resistance, whose value is strongly dependent on the quality of the contacts. For poor contacts this value may easily rise up to $100 \mathrm{k} \Omega$, even if recently the fabrication techinques are demonstrated very good quality contacts ( $\mathrm{Li}$ et al, 2009). We include these phenomena by adding two lumped resistors $R_{P} / 2$ in series to the line (see Fig.6b), taking into account both the bulk resistance and the imperfect contact resistance. 


\subsection{Circuit model for bundles of CNTs}

The huge value of the resistance makes useless an interconnect made by a single CNT shell. For practical application purposes, bundles of SWCNTs or MWCNTs are proposed as material to be used in fabricating interconnect traces (ITRS, 2007): all the CNT shells are fed in parallel, so lowering the total resistance. Following the stream of what done in paragraph 3.1, we can model the propagation along a CNT bundle in the frame of the multiconductor TL theory, starting from the scheme in Fig.8.

Let $\mathbf{V}(\omega, z)=\left[V_{1}(\omega, z) \ldots . V_{N}(\omega, z)\right]^{T}$ and $\mathbf{I}(\omega, z)=\left[I_{1}(\omega, z) \ldots I_{N}(\omega, z)\right]^{T}$ be the vectors of the $N$ voltages and currents at given $z$, in frequency domain. On the $k$-th CNT eq. (15) reads:

$$
\left(v_{k}+i \omega\right) I_{k}(\omega, z)+\frac{v_{F}^{2}}{\left(1+v_{k} / i \omega\right)} \frac{\partial Q_{k}(\omega, z)}{\partial z}=2 v_{F} \frac{M_{k}}{R_{0}} E_{z k}(\omega, z) .
$$

Let us assume that in evaluating the low-energy band structure we can neglect the interactions between the CNT shells, as shown by experimental evidence (Maarouf et al., 2000). This assumption allows using the results obtained for isolated CNT shells in evaluating the parameters $M_{k}$ and $v_{k}$ in (29). Using again (16)-(17) to derive $E_{z k}(\omega, z)$ and imposing the charge conservation law, we get the MTL equations:

$$
-\frac{d \mathbf{V}(\omega, z)}{d z}=(R+i \omega L) \mathbf{I}(\omega, z), \quad-\frac{d \mathbf{I}(\omega, z)}{d z}=i \omega \mathrm{CV}(\omega, z),
$$

with the pul parameter matrices given by:

$$
L=\alpha_{C}^{-1}\left(L_{M}+L_{k}\right), \quad C=C_{E}, \quad R=\alpha_{C}^{-1} L_{k} v, \quad \alpha_{C}=I+C_{q}^{-1} C_{e} ，
$$

where $I$ is the identity matrix, and the other matrices are given by:

$$
v=\operatorname{diag}\left(v_{k}\right), \quad L_{k}=\operatorname{diag}\left(L_{k k}\right), \quad C_{q}=\operatorname{diag}\left(C_{q k}\right) .
$$

(a)
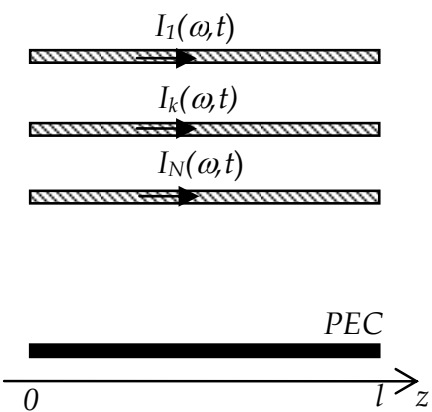

(b)

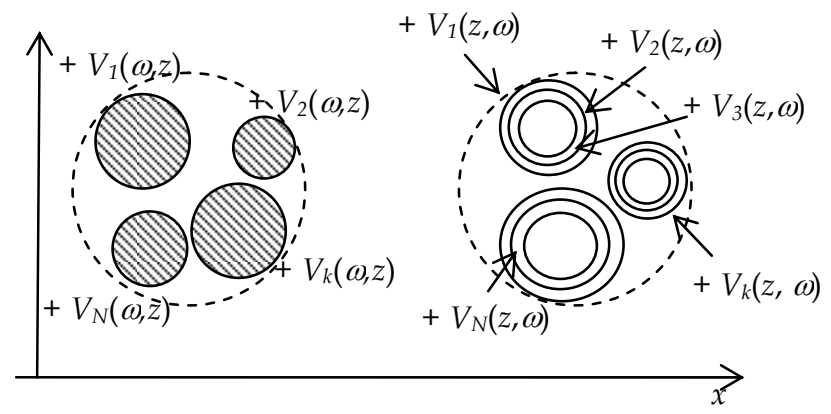

Fig. 8. A bundle of CNTs modeled as a multiconductor interconnect: (a) longitudinal view; transverse section of (b) SWCNTs and (c) MWCNTs bundle. 
In practical applications any CNT bundle is used to carry a single signal, hence a CNT bundle above a ground may be described by a two-conductor TL model, which could be rigorously derived from the MTL one (30)-(32) assuming all the CNTs in parallel. Alternatively, we can use approximated expressions for its pul parameters. It is important to stress that statistically a low-cost fabrication process for CNT bundles ends up with $1 / 3$ of metallic CNTs and 2/3 semiconducting ones (Li et al., 2005; Naeemi \& Meindl, 2006). In addition we have to recall that typical values of SWCNTs diameters fall in the range $2 \div 10 \mathrm{~nm}$ (Cheung et al., 2002), whereas the external shells of MWCNTs have typically diameters greater than $20 \mathrm{~nm}$ ( $\mathrm{Li}, 2005$; Naeemi, 2006). Recalling the results of Section 2, we can state that in a bundle of SWCNTs we may assume $1 / 3$ conducting $(M=2)$ and $2 / 3$ non conducting $(M=0)$, whereas all the MWCNTs shells may assumed to be conducting.

Let us first focus on the bundle capacitance. Figure 9 shows two circular bundles made by MWCNTs of external diameter $D_{\text {out }}=20 \mathrm{~nm}$, embbedded in a homogeneous dielectric matrix of $\mathrm{SiO}_{2}$ with $\varepsilon_{r}=4.5$. The bundle diameter is $D_{b}=146 \mathrm{~nm}$ and the center-center distance is $s=300 \mathrm{~nm}$. Figure 9 shows the distribution of the electrostatic potential, assuming a differential mode on the pair, which leads to a computed pul capacitance of $88.7 \mathrm{pF} / \mathrm{m}$. The classical pul capacitance of a pair of solid cylindrical conductors would be:

$$
C_{e}=\pi \varepsilon / \cosh ^{-1}\left(\frac{2 s}{D_{b}}\right)
$$

Using (33) would give a value of $92.8 \mathrm{pF} / \mathrm{m}$, with an error of less of $5 \%$. In a SWCNTs only $1 / 3$ of the SWCNTs are metallic, hence the distribution of the potential may sensibly vary assuming random distribution of the fraction of metallic CNTs in the bundle. Figure 10 shows two possible cases, referred to a pair made by two bundles of SWCNTs with CNT diameter $D=2 \mathrm{~nm}$, bundle diameter $D_{b}=18.8 \mathrm{~nm}$, center-center distance $s=38.2 \mathrm{~nm}$, embedded in a $\mathrm{SiO}_{2}$ matrix $\left(\varepsilon_{r}=4.5\right)$. Table 3 shows the results obtained for the cases of Fig.10 and assuming all the CNTs metallic (case 3). In this condition approximation (33) would give an error of $20 \%$. This approximation is acceptable or not depending on the values of the load capacitance in practical applications, as will be shown in Section 4.

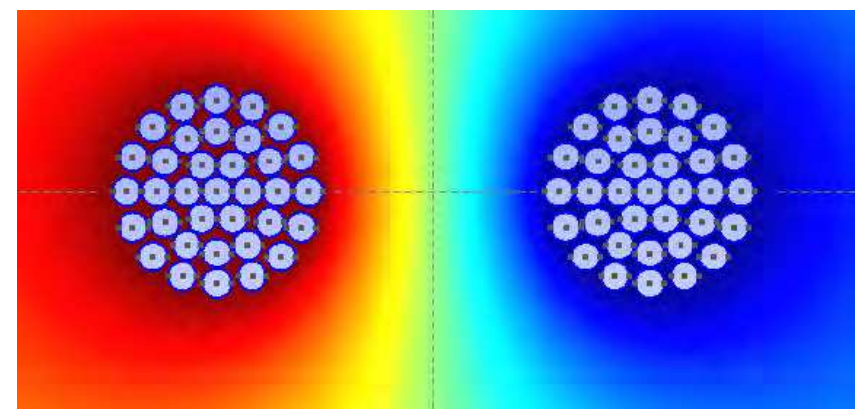

Fig. 9. Distribution of electrostatic potential for a differentially-fed pair of MWCNT bundles. 
(a)

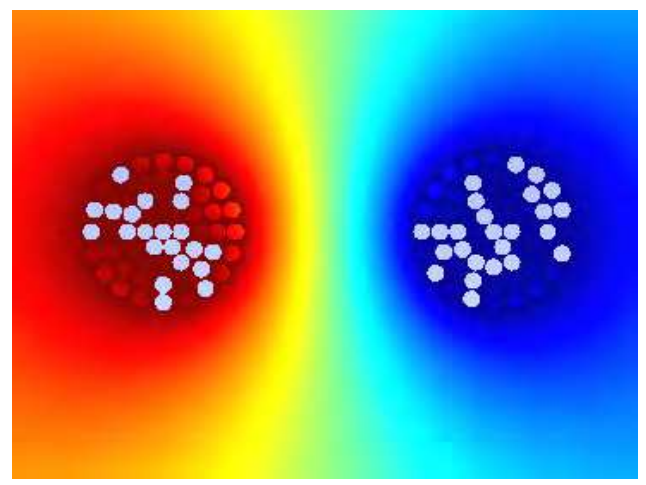

(b)

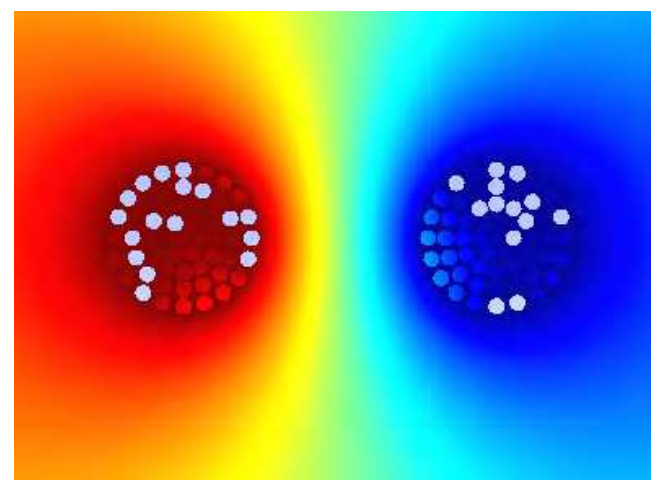

Fig. 10. Distribution of the electrostatic potential for a differentially-fed pair of SWCNT bundles: two random cases obtained assuming that $1 / 3$ of CNTs are metallic (highlighted).

\begin{tabular}{|c|c|c|c|}
\hline Case & $\begin{array}{c}C_{b} \text { (exact) } \\
{[\mathrm{pF} / \mathrm{m}]}\end{array}$ & $\begin{array}{c}C_{b}(33) \\
{[\mathrm{pF} / \mathrm{m}]}\end{array}$ & \% err \\
\hline 1 & 76.5 & 92.8 & 17.6 \\
\hline 2 & 74.3 & 92.8 & 19.9 \\
\hline 3 & 87.9 & 92.8 & 5.4 \\
\hline
\end{tabular}

Table 3. Evaluation of the bundle pul capacitance for the SWCNT cases depicted in Fig.10.

Let us now investigate the behavior of the bundle pul inductance and resistance. The magnetic inductance of the bundle may be computed from the vacuum space electrostatic capacitance, since it is $L_{m}=\mu_{0} \varepsilon_{0} C_{e 0}^{-1}$, where $C_{e 0}$ may be computed exactly or may be approximated by (33) if the introduced error is acceptable (which is the case of MWCNT bundles). When considering the kinetic inductance $L_{k b}$ of a bundle of N SWCNTs, we may assume that only $1 / 3$ are metallic, each of them contributing with $L_{k}=L_{k 0}$. In addition, if we consider CNTs with the same diameters, we have a unique value for $v$, hence:

$$
L_{k b}=\frac{L_{k 0}}{N / 3}, \quad R_{b}=\frac{v L_{k 0}}{N / 3}=\frac{3}{4} \frac{R_{0}}{l_{m f p} N} .
$$

Recalling the results in Table 2, for $N$ up to the order of $10^{3}$ we may still assume that $L_{k b} \gg L_{m}$, and since it is $\alpha_{C} \approx 1$, we may approximate the total bundle inductance with the first of (34), hence following the simple scaling law obtained in literature (Maffucci et al., 2009). Let us consider the case of a microstrip with effective dielectric constant $\varepsilon_{\text {reff }}=2.2$, where the signal trace of width $w=27 \mathrm{~nm}$, thickness $t=2 \mathrm{w}$ and distance to ground $2 t$ is made by a bundle of N SWCNTs of diameter $D=2.2 \mathrm{~nm}$. Figure 11 shows the self admittance of the line computed with the rigorous approach and with the proposed approximation, for $N=100$ and $N=200$. Here we have assumed $v_{F}=8.7 \cdot 10^{5} \mathrm{~m} / \mathrm{s}$ and $l_{m f p}=1 \mu \mathrm{m}$. 
(a)

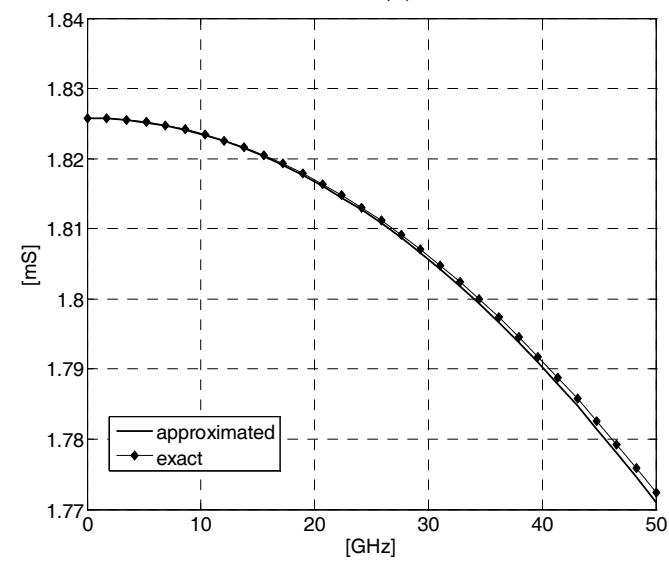

(b)

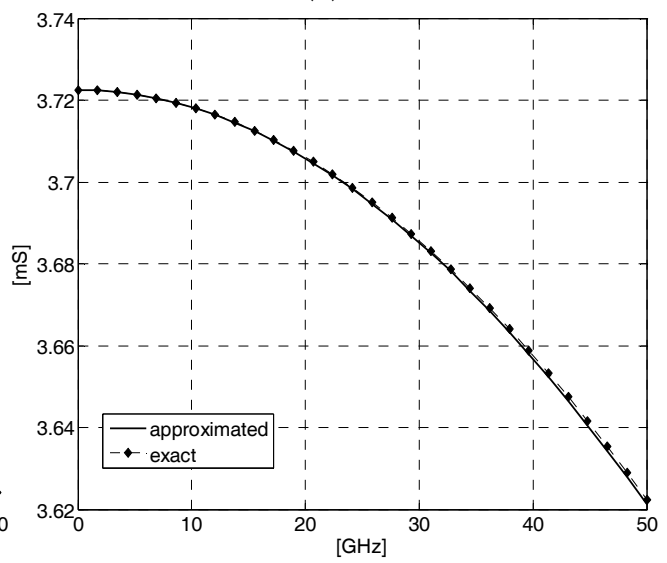

Fig. 11. Self admittance for a bundle of (a) 100 and (b) 200 SWCNTs.

Let us consider MWCNTs bundles. For these structures, the electrostatic capacitance and hence the magnetic inductance may be computed from (33). As for the kinetic inductance, we may introduce a simple approximation based on (13). Let $D_{\text {in }}$ and $D_{\text {out }}$ be the outer and inner shells diameters, respectively. The inter-shell spacing is the Van-der Waals distance $\delta=0.34 \mathrm{~nm}$ (Li et al., 2005), hence the number of shells is $N_{\text {shell }}=1+\left(D_{\text {out }}-D_{\text {in }}\right) / 2 \delta$. Once again we assume $1 / 3$ of the shells to be metallic and $2 / 3$ semiconducting. Assuming the above distribution and using approximation (13) we can compute the equivalent number of channels for a single shell of diameter $D_{s}$ in a MWCNTs through the simple law:

$$
M_{\text {shell }} \approx\left\{\begin{array}{lr}
2 / 3 & \text { for } D<d_{s} / T \\
a_{1 s} D_{s} T+a_{2 s} & \text { for } D \geq d_{s} / T
\end{array}\right.
$$

where $a_{1 s}=3.3 \cdot 10^{-4} 1 / \mathrm{nmK}, a_{2 s}=-0.08$ and $d_{s}=1.9 \cdot 10^{3} \mathrm{~nm} \cdot \mathrm{K}$. The kinetic inductance of a bundle of $N_{\mathrm{CNT}}$ MWCNTs is then given by:

$$
L_{k b} \approx \frac{2 L_{k 0}}{N_{C N T} N_{\text {shell }} M_{\text {shell }}} .
$$

where $L_{k 0}$ is the bulk value defined in (24). Using (36) in (21) we can derive the bundle quantum capacitance. Using $\alpha_{C} \approx 1$, from (19), (21) and (28) we may derive the bundle resistance:

$$
R_{b} \approx v L_{k b} \approx \frac{2 v L_{k 0}}{N_{C N T} N_{\text {shell }} M_{\text {shell }}}=\frac{1}{2 l_{\text {mfp }}} \frac{R_{0}}{N_{C N T} N_{\text {shell }} M_{\text {shell }}} .
$$

Note that the mean-free-path $l_{m f p}$ may be a function of the diameter $D$. A simple linear dependence is proposed in (Nieuwoudt \& Massoud, 2006). 


\section{Performance analysis of carbon nanotube interconnects}

This Section is devoted to performance analysis of CNT interconnects, compared to conventional $\mathrm{Cu}$ ones. The investigated scenarios refer to typical arrangements foreseen for future $22 \mathrm{~nm}$ technology node, expected for year 2016 and beyond (ITRS, 2007). In the following the circuit models for CNT interconnects are those presented in Section 3, whereas the conventional ones are described through classical TL models.

\subsection{Signal integrity analysis for CNT on-chip interconnects}

Let us consider the on-chip interconnect in Fig.12 made by two horizontal traces and a vertical via. The electrical and geometrical parameters given in Table 4 are typical values for the intermediate level at $22 \mathrm{~nm}$ technology node (ITRS, 2007). The signal traces on the layers and the via barrel may be either constituted by a solid $\mathrm{Cu}$ conductor or by a SWCNT bundle. In particular we consider the case of traces made by bundles of SWCNTs of $D=0.94 \mathrm{~nm}$, with a fraction of $1 / 3$ metallic. At the ends of the two horizontal tracts, a lumped contact resistance of $50 \mathrm{k} \Omega$ is considered for each metallic CNT. For the Cu case, the horizontal tracts are described as striplines, whereas the via is modeled through a series impedance $R-L$ and a pad capacitance to ground (e.g., Chiariello et al., 2009):

$$
R_{\text {via }}=\frac{\rho l_{\text {via }}}{\pi\left(d_{\text {via }} / 2\right)^{2}}, C_{\text {via }} \approx \frac{1.41 \varepsilon_{r} D_{P} T}{D_{A P}-D_{P}}[p F], L_{\text {via }} \approx 5.08 \cdot l_{\text {via }} \cdot\left[\ln \left(\frac{4 l_{\text {via }}}{d_{\text {via }}}\right)+1\right][n H],
$$

where $d_{\text {via }}$ and $l_{\text {via }}$ are the diameter and the length of the via barrel, respectively, $T$ is the dielectric thickness, $D_{P}$ and $D_{A P}$ are the diameters of the pad and the antipad, respectively.

(a)

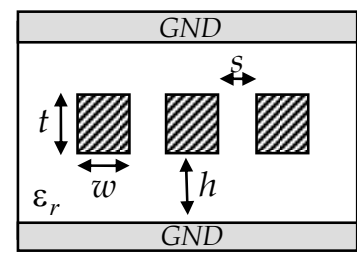

(b)

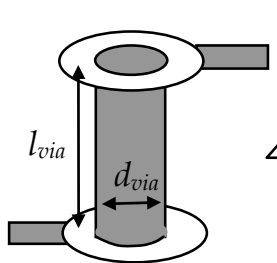

(c)

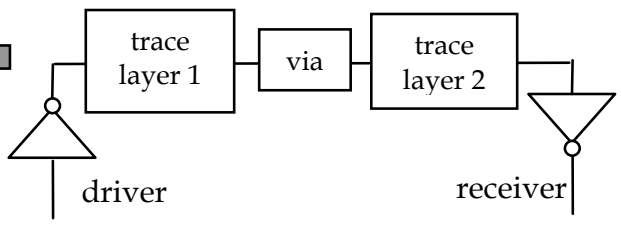

Fig. 12. On-chip interconnect: (a) section of the horizontal tracts; (b) vertical via; (c) circuit.

\begin{tabular}{|c|c|c|c|c|c|c|c|}
\hline $\begin{array}{c}w \\
{[\mathrm{~nm}]}\end{array}$ & $\begin{array}{c}t \\
{[\mathrm{~nm}]}\end{array}$ & $\begin{array}{c}h \\
{[\mathrm{~nm}]}\end{array}$ & $\begin{array}{c}s \\
{[\mathrm{~nm}]}\end{array}$ & $\begin{array}{c}d_{\text {via }} \\
{[\mathrm{nm}]}\end{array}$ & $\begin{array}{c}l_{\text {via }} \\
{[\mu \mathrm{m}]}\end{array}$ & $\begin{array}{c}\varepsilon_{\mathrm{r}} \\
{\left[\mathrm{SiO}_{2}\right]}\end{array}$ & $\begin{array}{c}\rho_{\mathrm{Cu}} \\
{[\mu \Omega \cdot \mathrm{cm}]}\end{array}$ \\
\hline 44 & 44 & 39.6 & 44 & 60 & 1.0 & 4.5 & 4.235 \\
\hline
\end{tabular}

Table 4. Parameters for the case study in Fig.12.

\begin{tabular}{|c|c|c|c|c|c|}
\hline & $\begin{array}{c}R \\
{[\Omega / \mu \mathrm{m}]}\end{array}$ & $\begin{array}{c}L \\
{[\mathrm{pH} / \mu \mathrm{m}]}\end{array}$ & $\begin{array}{c}C \\
{[\mathrm{fF} / \mu \mathrm{m}]}\end{array}$ & $\begin{array}{c}R_{\text {via }} \\
{[\Omega]}\end{array}$ & $\begin{array}{c}L_{\text {via }} \\
{[\mathrm{pH}]}\end{array}$ \\
\hline $\mathrm{Cu}$ & 22.31 & 0.30 & 0.17 & 10.19 & 0.03 \\
\hline $\mathrm{CNT}$ & 2.29 & 2.62 & 0.17 & 1.29 & 1.47 \\
\hline
\end{tabular}

Table 5. Computed pul and lumped parameters. 
As for the terminations in Fig.12c, we assume that the driver is modeled as a voltage source of $V_{s}=0.8 V_{D D}$ and a series resistor of $1.5 \mathrm{k} \Omega$, whereas the receiver is modeled as a capacitor of $1 \mathrm{fF}$. These are typical values for the 22-nm node, obtained from the minimum size gate values in the case with a size factor of $20 x$ ( $\mathrm{Li}$ et al., 2009).

Let us investigate the signal integrity performances of these two systems, assuming an high data rate (DR) signal transmission. Figure 13 shows the eye-diagrams obtained for the $\mathrm{Cu}$ case (Fig.13a-b) and the CNT case (Fig.13c-d), for values of the data rate DR ranging from 3 to $20 \mathrm{Gbit} / \mathrm{s}$. The complete SI analysis is given in Table 6. The jitter is of the same order, whereas the performances of CNTs in terms of eye-opening factor are much better than the $\mathrm{Cu}$ ones. For instance at $10 \mathrm{Gbit} / \mathrm{s}$ the $\mathrm{Cu}$ channel could not be used (Fig.13b), whereas the CNT one still shows good performances (Fig.13c). The behavior obtained here is justified from the consideration that the higher inductance of CNT is compensated by its lower resistance and the SI performance of the overall CNT channel is better than $\mathrm{Cu}$.

(a)

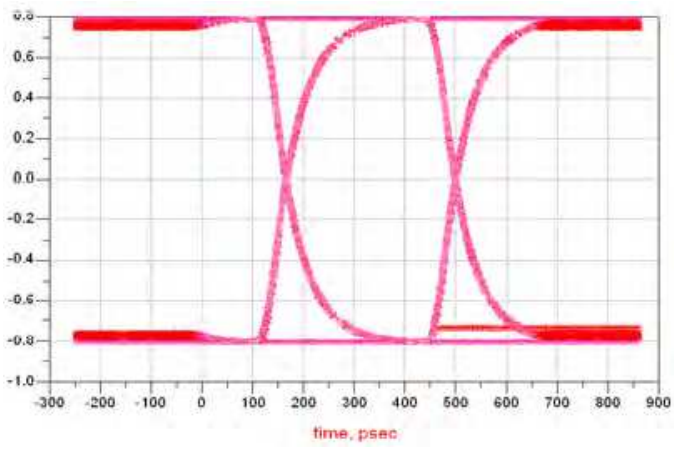

(c)

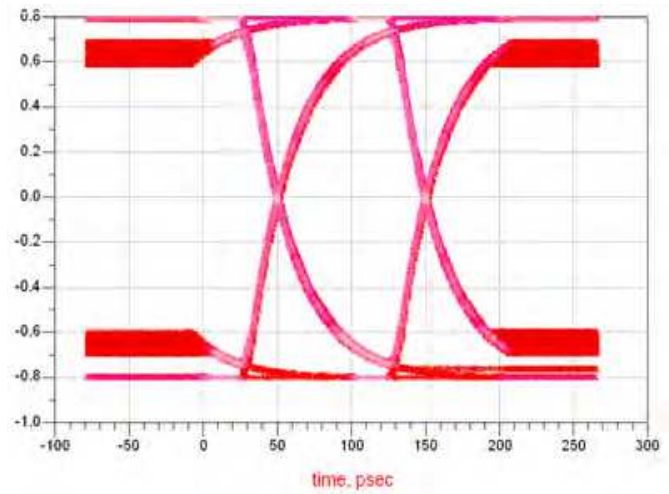

(b)

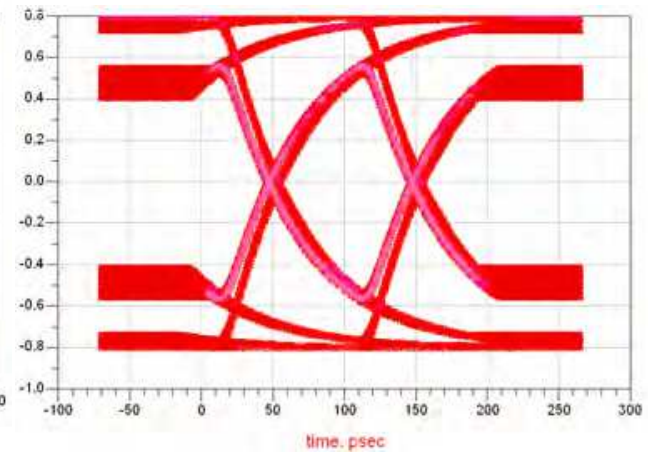

(d)

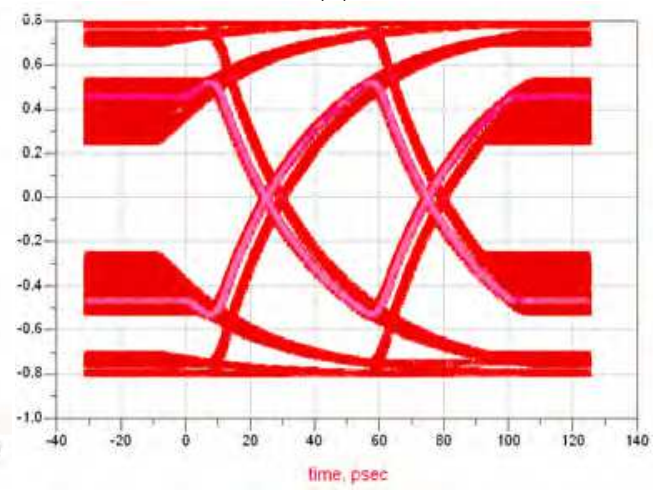

Fig. 13. Eye-diagram analysis for the case in Fig.12: Cu interconnect with (a) $D R=3 \mathrm{Gbit} / \mathrm{s}$, $t_{\mathrm{r}}=33 \mathrm{ps}$; and (b) $D R=10 \mathrm{Gbit} / \mathrm{s}, t_{\mathrm{r}}=10 \mathrm{ps} ; \mathrm{CNT}$ interconnect with (c) $D R=10 \mathrm{Gbit} / \mathrm{s}, t_{\mathrm{r}}=10 \mathrm{ps}$; and (d) $D R=20 \mathrm{Gbit} / \mathrm{s}, t_{\mathrm{r}}=5 \mathrm{ps}$. 


\begin{tabular}{|c|c|c|c|c|}
\hline & $\begin{array}{c}D R \\
{[\mathrm{Gbit} / \mathrm{s}]}\end{array}$ & $\begin{array}{c}t_{\mathrm{r}} \\
{[\mathrm{ps}]}\end{array}$ & $\begin{array}{c}\text { Eye jitter } \\
{[\mathrm{ps}-\mathrm{rms}]}\end{array}$ & $\begin{array}{c}\text { Eye opening } \\
\text { factor }\end{array}$ \\
\hline $\mathrm{CNT}$ & 1 & 100 & 2.1 & 1 \\
\hline $\mathrm{Cu}$ & 1 & 100 & 1.6 & 1 \\
\hline $\mathrm{CNT}$ & 3 & 33 & 166 & 0.99 \\
\hline $\mathrm{Cu}$ & 3 & 33 & 167 & 0.97 \\
\hline $\mathrm{CNT}$ & 5 & 20 & 100 & 0.98 \\
\hline $\mathrm{Cu}$ & 5 & 20 & 100 & 0.91 \\
\hline $\mathrm{CNT}$ & 10 & 10 & 50 & 0.89 \\
\hline $\mathrm{Cu}$ & 10 & 10 & 50 & 0.76 \\
\hline $\mathrm{CNT}$ & 20 & 5 & 24 & 0.72 \\
\hline $\mathrm{Cu}$ & 20 & 5 & 22 & 0.47 \\
\hline
\end{tabular}

Table 6. Results of the Eye-Diagram analysis for the case-study in Fig.12.

\subsection{EMC behavior of CNT interconnects}

As pointed out in Section 3, the parameters of the circuit model for CNT interconnects are strongly affected by the presence of a kinetic inductance and a quantum capacitance. Within the limits of the model used here, these two parameters are independent on frequency, hence the frequency behavior of CNT interconnects may be completely different from that of conventional ones. Here we investigate two typical problems arising when performing an electromagnetic compatibility (EMC) analysis: the high-frequency effects on the line impedance and the crosstalk noise.

The case-study used for EMC analysis is the stripline configuration in Fig.14a, assuming global level parameters: $w=0.32 \mu \mathrm{m}, t=0.19 \mu \mathrm{m}, h=0.15 \mu \mathrm{m}$ and $\varepsilon_{r}=2.2$. Three different realizations of the signal traces are compared: solid copper, a bundle of SWCNTs and a bundle of MWCNTs. At such a scale the copper resistivity is $\rho \approx 2.942 \mu \Omega \cdot \mathrm{cm}$.

Let us first study the high-frequency impedance. The first realization of the signal traces is made a bundle of SWCNTs, of diameter $D=1.4 \mathrm{~nm}$, assuming the area of the trace filled by CNTs with a density of $80 \%$, and considering $1 / 3$ of the total number of CNTs to be metallic. The second realization is made by a bundle of MWCNTs, with the same density, each of them with outer shell diameter of $D_{\text {out }}=40 \mathrm{~nm}$, inner shell diameter $D_{\text {in }}=0.5 D_{\text {out }}$ and inter-shell distance $\delta=0.34 \mathrm{~nm}$. The temperature is $300 \mathrm{~K}$.

(a)

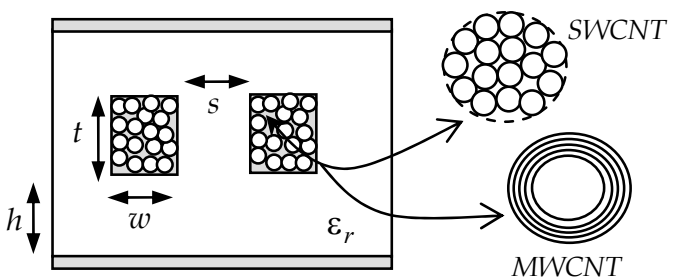

(b)

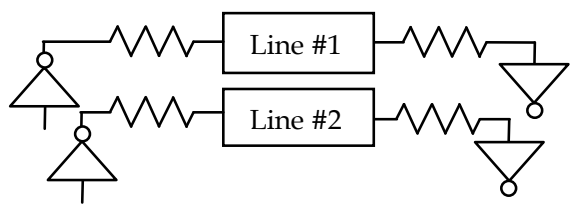

Fig. 14. Interconnect geometry used for EMC analysis: (a) the signal traces are made by either solid $\mathrm{Cu}$ or CNT bundles (SWCNT or MWCNTs); (b) circuit for the crosstalk analysis. 
The p.u.l. resistances for the three realizations are reported in Fig.15a. For $\mathrm{Cu}$ lines this parameter is affected by the skin-effect, whereas for CNTs this effect is not present. For instance, at $200 \mathrm{GHz}$ the penetration depth is $0.19 \mu \mathrm{m}$, equal to $t$ and lower than $w$. The $\mathrm{CNT}$ interconnects outperform the $\mathrm{Cu}$ one and show a resistance parameter insensitive to the skin effect. In particular, the MWCNTs bundle outperforms the other two realizations in the whole frequency range: this is due to the fact that all the shells contribute to the conduction. As for the p.u.l. inductance (Fig.15b), the $\mathrm{Cu}$ line shows a slight decrease of this parameters, due to the decrease of the contribution of the internal inductance with increasing frequency, whereas the CNTs bundles are again insensitive to it. The MWCNT bundle interconnect introduces an inductance comparable to the $\mathrm{Cu}$ one, whereas the SWCNT realization shows an higher inductance. Since the kinetic inductance of the bundles decreases as (34) or (36), it may be comparable to the magnetic one, as in this case. As a conclusion we may assume that CNT interconnects exhibit parameters independent on frequency, whereas the conventional ones would suffer from this problem.

For the crosstalk analysis we consider a coupled stripline as in Fig.14a, assuming $s=w$, and typical intermediate level parameters: $w=t=44 \mathrm{~nm}, h=39.6 \mathrm{~nm}$ and $\varepsilon_{r}=2.2$. The considered circuit is shown in Fig.14b. We assume all the resistors of $1450 \Omega$ assuming that line 1 is fed at near end by a time-harmonic voltage source of amplitude $V_{\text {in }}=1$ (a.u.). The far-end receivers are modeled as capacitors of $1 \mathrm{fF}$. For the CNT realizations we consider a density of $80 \%$, a temperature of $300 \mathrm{~K}$ and a mean free path $l_{m f p}=0.5 \mu \mathrm{m}$. The SWCNTs diameter is $D=1.4 \mathrm{~nm}$, whereas for the MWCNTs the outer shell diameter is $D_{\text {out }}=30 \mathrm{~nm}$, the inner shell diameter is $D_{\text {in }}=0.5 D_{\text {out }}$ and the inter-shell distance is $\delta=0.34 \mathrm{~nm}$. Because of the large number of CNTs in the bundle, we may disregard the effect of the parasitic contact resistances. Figure 16 shows the computed near-end and far-end crosstalk voltages, normalized to the input voltage $V_{\text {in }}$. The crosstalk noise for the three realizations is of the same order, hence from this point of view we may assume an equivalent EMC performance.

(a)

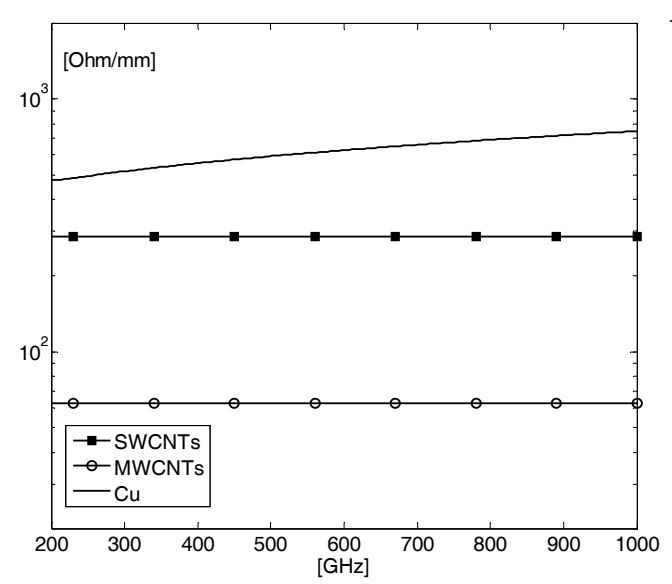

(b)

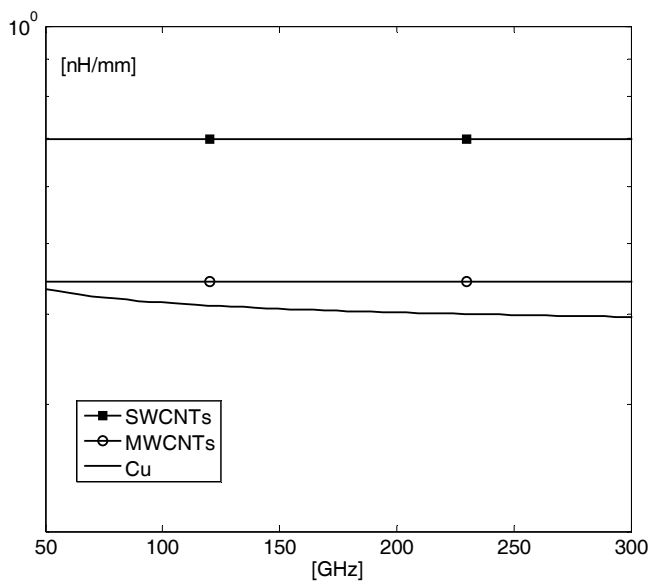

Fig. 15. (a) P.u.l. resistance for a trace made by $\mathrm{Cu}$, a bundle of SWCNTs or MWCNTs;

(b) P.u.l. inductance for a trace made by $\mathrm{Cu}$, a bundle of SWCNTs or MWCNTs. 
(a)

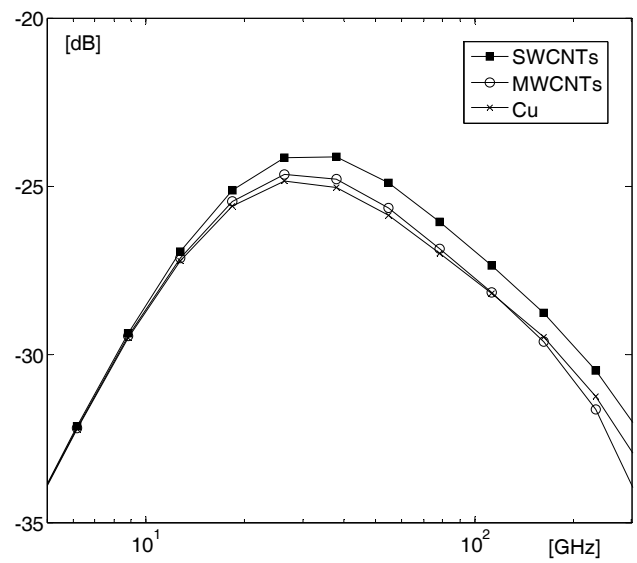

(b)

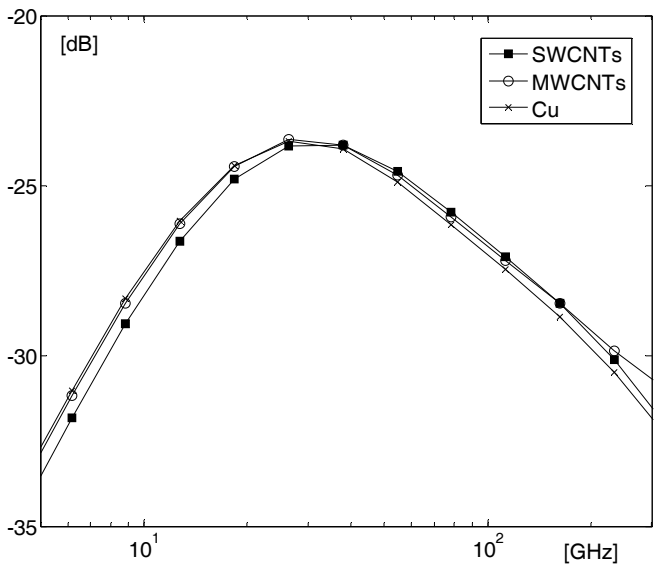

Fig. 16. Crosstalk level for a coupled line made by copper trace, SWCNTs bundle and MWCNTs bundle: (a) near end; (b) far end.

\subsection{CNT interconnects as pillars for nanopackaging}

Recently the CNTs have been proposed as material for nanopackaging interconnects, since their unique electrical, mechanical and thermal properties allows meeting many of the new requirements for bonding, molding compound, underfill, thermal interface, die attach (Morris, 2008; Maffucci, 2009). One of the main reasons pushing towards the use of CNTs in nanopackaging is the possibility either to achieve good electrical performances and implement new heat removal technologies. The thermal management of future nanoelectronics requires new approaches, since the classical heat removal techniques are inadequate. Using the conventional approach, for instance, the cooler for future Systems in Package would require much greater volume than the semiconductor itself. Recently, CNTs have been proposed as microchannel coolers in thermofluidic cooling approaches and as thermal interface material (TIM). The main limit to the practical use of CNTs is still given by the possibility of integrating a high density of aligned CNTs in a polymer matrix, without degrading thermal conductivity.

In order to investigate the electrical properties of CNT nanopackaging interconnects, let us consider the use of CNTs bundles as chip to package interconnect, following the scheme depicted in Fig.17a: the two vertical pillars are realized using $\mathrm{Cu}$, a bundle of SWCNTs (Fig.17b) or a bundle of MWCNTs (Fig.17c). In terms of electrical properties, the goal of a good packaging interconnect is of course to introduce low parasitics.

As done before, in the following we assume that in the SWCNT bundle only a fraction of $1 / 3$ of the total CNTs is metallic, and similarly in a MWCNT only $1 / 3$ shells are metallic. Using the results in Section 2, since the semiconducting SWCNTs do not give contribution to the conduction, very high density bundles must be fabricated to have low resistance CNT pillars. In MWCNTs, instead, the semiconducting shells give a non-negligible contribution to the conduction and the conduction of the metallic shells themselves is improved, hence the optimal density is a trade-off between the MWCNT outer diameter and its shell number. 
(a)

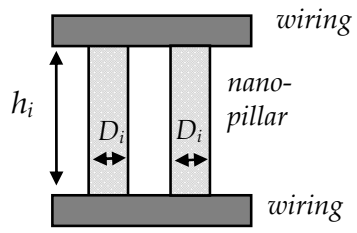

(b)

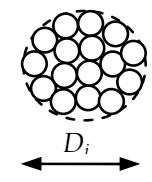

SWCNT (c)

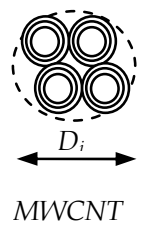

Fig. 17. Chip-to-package interconnect: (a) scheme; (b) SWCNTs and (c) MWCNTs realization

(a)

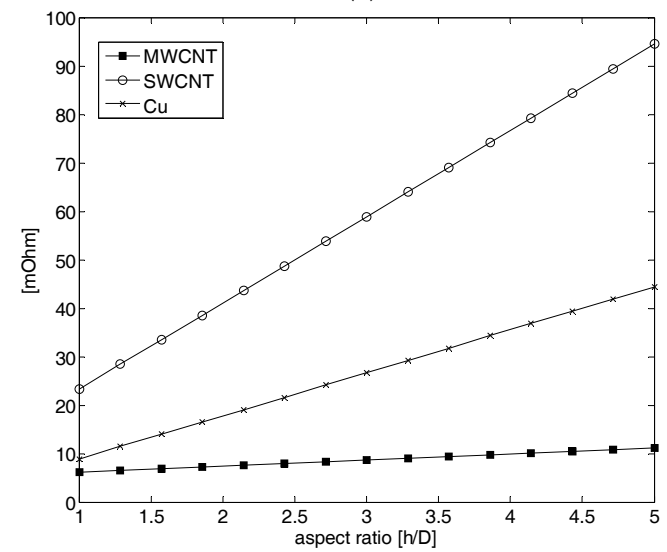

(b)

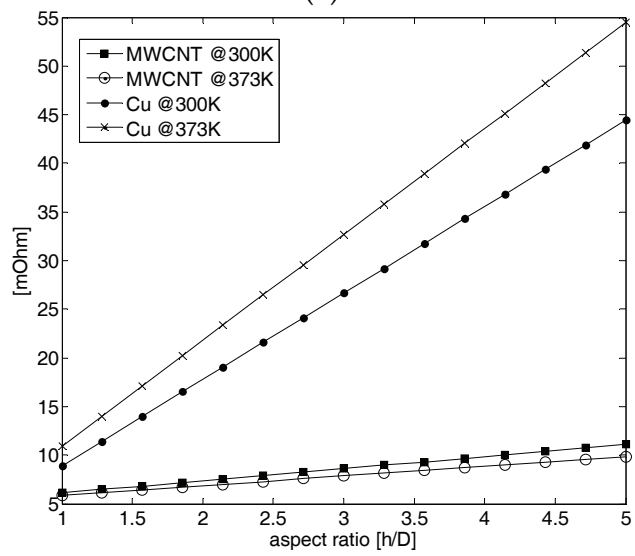

Fig. 18. Parasitic resistance: (a) vs aspect ratio, at $\mathrm{T}=300 \mathrm{~K}$; (b) vs temperature.

Let us consider the case of Fig. 17, assuming the pillars to be cylinders of diameter $D_{i}$ and height $h_{i}$, a wire bond pitch of $20 \mu \mathrm{m}$ and an operating frequency of $10 \mathrm{GHz}$ at a temperature of $300 \mathrm{~K}$. First we consider $\mathrm{Cu}$ interconnects with $\sigma_{\mathrm{Cu}}=5.8 \cdot 10^{7} \mathrm{~S} / \mathrm{m}$. Next, we fill the interconnect with a bundle of SWCNTs of mean diameter $2.7 \mathrm{~nm}$, assuming a density of $80 \%$ of the total area and assuming $1 / 3$ of metallic CNTs. In addition, a parasitic contact resistance of $20 \mathrm{k} \Omega$ in series to any single CNT is considered. Finally we consider the bundle to be filled by MWCNTs with outer diameter $D_{\text {out }}=30 \mathrm{~nm}$, inner diameter $D_{\text {in }}=0.5 D_{\text {out }}$ and inter-shell distance $0.34 \mathrm{~nm}$. We assume again $1 / 3$ of metallic shells and the rest semiconducting, a density of $80 \%$ and a contact resistance of $10 \mathrm{k} \Omega$ per shell. The considered mean free path for all CNTs is $l_{m f p}=0.5 \mu \mathrm{m}$.

For this case the parasitic inductances are well beyond the maximum allowed of $5-10 \mathrm{pH}$, hence we focus on the resistance. Figure 18a compares the resistance values obtained for aspect ratios ranging from 1 to 5 . The lowest values are obtained by MWCNT bundle, whereas SWCNT bundle shows higher resistance. The better performance of MWCNTs is due to the contribution to the conduction given by the semiconducting shells.

Let us now investigate the problem of the compatibility of CNT interconnects with the temperatures of CMOS technology. Let us consider a realistic chip operating temperature of 
$\mathrm{T}=373 \mathrm{~K}$. When the temperature increases the kinetic inductance decreases, as shown in Section 2, and the difference is more pronounced for large diameters, hence for MWCNTs rather than for SWCNTs. Let us neglect the variation of the mean-free path with temperature: in the same conditions described above, the parasitic resistance for the MWCNT bundle and the $\mathrm{Cu}$ interconnects would be that shown in Fig.18b. Note that for the copper at $373 \mathrm{~K}$ we have assumed $\sigma_{\mathrm{Cu}}=4.0 \cdot 10^{7} \mathrm{~S} / \mathrm{m}$. The MWCNT interconnect has better performances at higher frequencies, which is opposite to the behaviour of $\mathrm{Cu}$ ones.

Finally let us focus on the problem of proximity effect, which is a major problem for packaging interconnect, since it leads to non uniform current crowding which cause local thermal hot-spots. Figure 19 shows the distribution of the current density over two CNT bundles at a distance equal to their width. The coupled line is driven by a differential signal (in the figures the underlying PEC ground is omitted). The simulations have been carried out by means of the full-wave 3D numerical simulator SURFCODE (Miano \& Villone, 2005). As shown in Figs.19a-b, the CNT bundles do not suffer from the proximity effect, which is a problem for the conventional interconnects, Figs.18c-d. This is again due to the presence in the CNT electrodynamics of a kinetic term which dominates over the magnetic one. Within the limits of validity of our model, this kinetic term is independent from the distance between the bundles, which, on the contrary, is a crucial parameter in determining the proximity effect when conventional interconnects are analyzed.

(a)

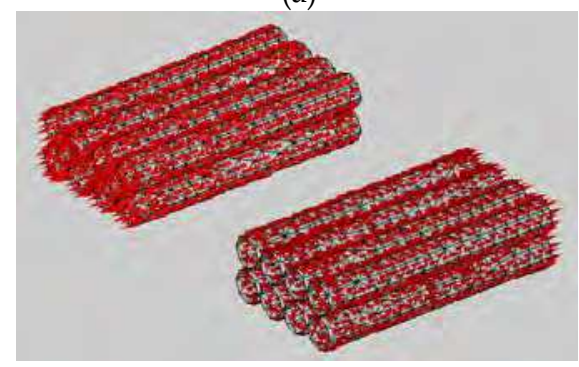

(c)

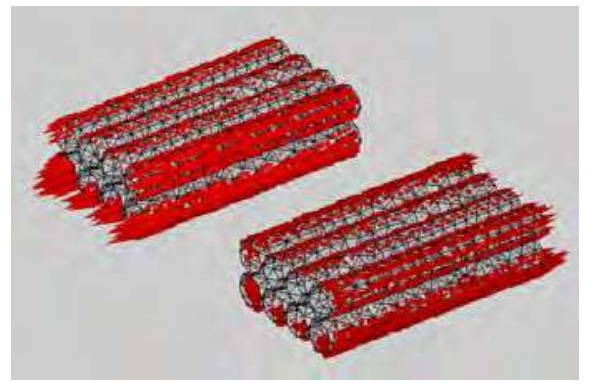

(b)

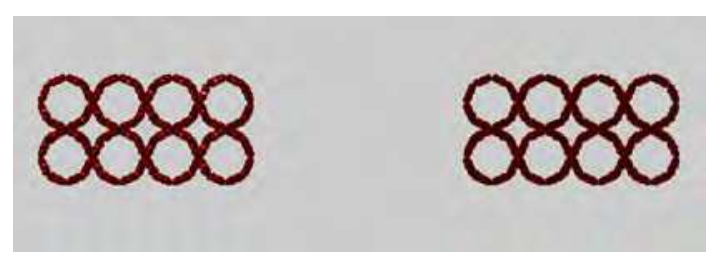

(d)

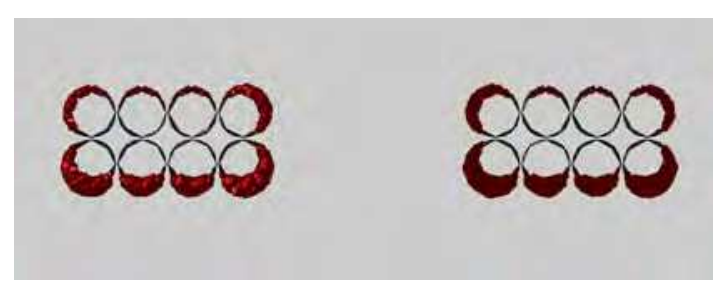

Fig. 19. Current density distributions over two bundles (differential mode): (a) CNT bundles; (b) CNT transverse section; (c) PEC bundles; (d) PEC transverse section. 


\section{Conclusion}

This Chapter has analyzed the use of carbon nanotubes as possible innovative material to fabricate high-speed interconnects at various hierarchical levels of the future nanoelectronics technology.

The electromagnetic model presented here to describe the electrical propagation along CNT interconnects is derived from a semi-classical solution of the transport equation. All the quantistic and kinetic effects affecting the electrodynamics of charge carriers are taken into account through global parameters (kinetic inductance and quantum capacitance) which have been simply related to the number of effective conducting channel per $\mathrm{CNT}$ shell. Both the rigorous approach and a simple approximation have been provided to evaluate this number for CNT shells of arbitrary chirality, hence allowing the modelling of either single-wall and multi-wall CNTs.

The electrodynamical model provides a constitutive equation for the CNT interconnect, which is coupled to the Maxwell equation: the problem is then solved in the frame of the classical transmission line theory, so obtaining simple equivalent RLC circuits for single CNTs and bundles of CNTs. These models are consistent with those used in literature for metallic CNTs, but introduce the possibility to deal with arbitrary chiralities and CNT diameters. The analysis of CNT transmission lines highlights unusual behaviours with respect to the $\mathrm{Cu}$ lines, mainly due to the strong influence of the kinetic and quantistic terms over the classical electromagnetic inductance and capacitance parameters.

Some case-studies have been carried out referring to a realistic use of CNTs to build interconnects for wiring and for packaging nanotechnology ICs. In the considered examples typical values of the future $22 \mathrm{~nm}$ technology nodes have been assumed. A signal integrity analysis is performed with reference to an on-chip interconnect arrangement, where the conventional $\mathrm{Cu}$ material is replaced by a bundle of CNTs. Next, the EMC behaviour of two adjacent traces in a stripline is analyzed, checking the high-frequency effects and the crosstalk noise. Finally the use of CNTs as pillars for nanopackaging is studied and the introduced parasitics are compared to conventional material realization.

Carbon nanotube interconnects are shown to have better behaviour with respect to conventional $\mathrm{Cu}$ ones in terms of SI and EMC performances, introduced parasitics and response to temperature variations. From a practical point of view, these simulated performances may be achieved provided that good quality bundles are realized, i.e. bundles of high density, good direction control and good terminal contacts.

\section{References}

Anantram, M.P.; \& Léonard, F. (2006). Physics of carbon nanotube electronic devices. Report Progress in Physics, Vol.69, 507.

Avouris, P.; Appenzeller, J.; Marte, R. \& Wind, S.J. (2003). Carbon nanotube electronics, Proceedings of IEEE, Vol.91, 1772.

Banerjee, K.; Li, H. \& Srivastava, N. (2008). Current status and future perspectives of carbon nanotubes interconnects. Proc. of IEEE Nanotechonology Conference, Arlington, TX, USA, August 18-21, 2008.

Burke, P.J. (2002). Luttinger liquid theory as a model of the gigahertz electrical properties of carbon nanotubes, IEEE Transactions on Nanotechnology, Vol. 1, 129-144. 
Burke, P.J. (2003). An RF circuit model for carbon nanotubes. IEEE Transactions on Nanotechnology, Vol.2, 55.

Cheung, C.L. \& et al. (2002). Diameter-controlled Synthesis of Carbon nanotubes. Journ. Phys. Chem. B, Vol.106, 2429-2433.

Chiariello, A.G.; Maffucci, A. \& Miano, G. (2009). Signal Integrity Analysis of Carbon Nanotube on-chip Interconnects. IEEE Workshop on Signal Propagation on Interconnects SPI 2009, paper no. S4_1, Strasbourg, France, 12-15 may 2009.

Close, G.F.; Yasuda, S.; Paul, B.; Fujita, S. \& Philip Wong, H.-S. (2008). A 1 GHz integrated circuit with carbon nanotube interconnects and silicon transistors. Nano Letters, Vol.8, 706-709.

Forestiere, C.; Maffucci, A. \& Miano, G. (2009a). A Generalized Model for the Signal Propagation along Single- and Multi-Walled Carbon Nanotubes with Arbitrary Chirality, Proc. of 9th IEEE Nanotechnology Conference, paper \#64, Genova (Italy), 2730 Jul 2009.

Forestiere, C.; Maffucci, A.; Miano, G., Maksimenko, S.A. \& Slepyan, G.Y. (2009b). Signal propagation in carbon nanotubes of arbitrary chirality. In press on IEEE Trans. On Nanotechnology.

ITRS (2007) International Technology Roadmap for Semiconductors. http://public.itrs.net. Edition 2007.

Iijima, S. (1991). Helical microtubules of graphitic carbon. Nature, Vol.354, 56-58.

Li H.J.; Lu, W. G.; Li, J. J. ; Bai, X. D. \& Gu, C. Z. (2005). Multichannel Ballistic Transport in Multiwall Carbon Nanotubes. Physical Review Letters, Vol.95, 086601.

Li, H.; Xu, C; Srivastava, N. \& Banerjee, K. (2009). Carbon Nanomaterials for Next Generation Interconnects and Passives: Physics, Status, and Prospects. IEEE Transactions on Electron Devices, Vol. 56, 1799-1821.

Liu, Z. (2009). Fabrication and electrical characterization of densified carbon nanotube micropillars for IC interconnection. IEEE Trans. on Nanotechnology, Vol.8, 196-203.

Maarouf, A.A.; Kane, C.L. \& Mele, E.J. (2000). Electronic structure of carbon nanotube ropes. Physical Review B, Vol.61, 11156-11165.

Maksimenko, S.A. \& Slepyan, G.Y. (2000). Electrodynamic properties of carbon nanotubes, in Electromagnetic Fields in Unconventional Materials and Structures, O. N. Singh and A. Lakhtakia Eds., pp. 217-255, Wiley, New York.

Maksimenko, S.A.; Khrushchinsky, A.A.; Slepyan, G.Y. \& Kibisb, O.V. (2007). Electrodynamics of chiral carbon nanotubes in the helical parametrization scheme. Journal of Nanophotonics, Vol.1, p.013505.

Maffucci A.; Miano, G. \& Villone, F. (2008a). A transmission line model for metallic carbon nanotube interconnects, Intern. Journal of Circuit Theory and Applic., Vol. 36, 31-52.

Maffucci A.; Miano, G. \& Villone, F. (2008b). Performance Comparison Between Metallic Carbon Nanotube and Copper Nano-Interconnects, IEEE Transactions on Advanced Packaging, Vol. 31, 692 - 699.

Maffucci A.; Miano, G. \& Villone, F. (2009). A new Circuit Model for Carbon Nanotube Interconnects with Diameter-dependent Parameters. IEEE Transactions on Nanotechnology, Vol.8, 345 - 354.

Maffucci, A. (2009). Carbon Nanotubes in Nanopackaging applications. IEEE Magazine on Nanotechnology, Vol.3, no.3, 22-25. 
Miano, G. \& Villone, F. (2005). A surface integral formulation of Maxwell equations for topologically complex conducting domains. IEEE Trans. on Antennas and Propagation, vol. 53, 4001-4014.

Miano, G. \& Villone, F. (2006). An Integral Formulation for the Electrodynamics of Metallic Carbon Nanotubes Based on a Fluid Model, IEEE Transactions on Antennas and Propagation, Vol.54, 2713-2724, 2006.

Morris, J.E. (2008). Nanopackaging: Nanotechnologies and electronics packaging. New-York: Springer.

Naeemi, A. \& Meindl, J. D. (2006). Compact Physical Models for Multiwall CarbonNanotube Interconnects. IEEE Electron Devices Letters, Vol.27, 338-340.

Naeemi, A. \& Meindl, J. D. (2008). Performance Modeling for Single- and Multiwall Carbon Nanotubes as Signal and Power Interconnects in Gigascale Systems, IEEE Trans. on Electron Devices, Vol. 55, n.10, 2574-2582.

Nieuwoudt, A. \& Massoud,Y. (2006). Evaluating the impact of resistance in carbon nanotube bundles for VLSI interconnect using diameter-dependent modeling techniques. IEEE Trans. Electron Devices, vol. 53, 2460-2466.

Park, J.Y.; et al., (2004). Electron-phonon Scattering in Metallic Single-Walled Carbon Nanotubes. Nano Letters, Vol.4, 517-520.

Raychowdhury, A.; Roy, K. (2006). Modelling of metallic carbon-nanotube interconnects for circuit simulations and a comparison with $\mathrm{Cu}$ interconnects for scaled technologies. IEEE Trans. on Computer-Aided Design for Integrated Circ. and Syst., Vol. 25, 58-65.

Rutherglen, C. \& Burke, P.J. (2009). Nanoelectromagnetics: Circuit and Electromagnetic Properties of Carbon Nanotubes. Small, Vol.5, 884-906.

Saito, R.; Dresselhaus, G. \& Dresselhaus, M. S. (2004). Physical Properties of Carbon Nanotubes, Imperial College Press, Singapore.

Salahuddin, S.; Lundstrom, M. \& Datta, S. (2005). Transport effects on signal propagation in quantum wires. IEEE Transactions on Electron Devices, Vol.52, 1734-1742.

Sarto, M.S.; Tamburrano, A. \& D'Amore, M. (2009). New Electron-Waveguide-Based Modeling for Carbon Nanotube Interconnects. IEEE Transactions on Nanotechnology, Vol. 8, 214-225.

Slepyan, G.Y.; Maksimenko, S. A.; Lakhtakia, A.; Yevtushenko, O. \& Gusakov, A.V. (1999). Electrodynamics of carbon nanotubes: dynamics conductivity, impedance boundary conditions, and surface wave propagation. Physical Review B, Vol. 60, 17136.

Soga, I.; Kondo, D.; Yamaguchi, Y.; Iwai, T.; Mizukoshi, M.; Awano, Y.; Yube, K. \& Fujii T. (2008). Carbon nanotube bumps for LSI interconnect, Proc. Of Electronic Components and Technology Conf., 1390-1394, May 2008.

Wesström, J.J. (1996). Signal propagation in electron waveguides: Transmission-line analogies, Physical Review B, Vol. 54, 11484-11491.

Pu, S-N.; Yin, W.-Y.; Mao, J.-F. \& Liu, Q.H. (2009). Crosstalk Prediction of Single- and Double-Walled Carbon-Nanotube (SWCNT/DWCNT) Bundle Interconnects. IEEE Transactions on Electron Devices, Vol. 56, 560-568. 


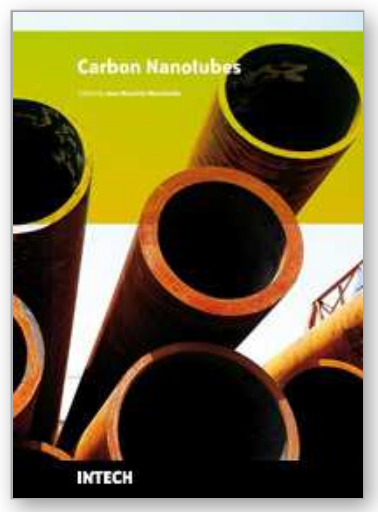

\author{
Carbon Nanotubes \\ Edited by Jose Mauricio Marulanda
}

ISBN 978-953-307-054-4

Hard cover, 766 pages

Publisher InTech

Published online 01, March, 2010

Published in print edition March, 2010

This book has been outlined as follows: A review on the literature and increasing research interests in the field of carbon nanotubes. Fabrication techniques followed by an analysis on the physical properties of carbon nanotubes. The device physics of implemented carbon nanotubes applications along with proposed models in an effort to describe their behavior in circuits and interconnects. And ultimately, the book pursues a significant amount of work in applications of carbon nanotubes in sensors, nanoparticles and nanostructures, and biotechnology. Readers of this book should have a strong background on physical electronics and semiconductor device physics. Philanthropists and readers with strong background in quantum transport physics and semiconductors materials could definitely benefit from the results presented in the chapters of this book. Especially, those with research interests in the areas of nanoparticles and nanotechnology.

\title{
How to reference
}

In order to correctly reference this scholarly work, feel free to copy and paste the following:

A.G. Chiarello, A. Maffucci, G. Miano and F. Villone (2010). Carbon Nanotubes Interconnects for Nanoelectronics Circuits, Carbon Nanotubes, Jose Mauricio Marulanda (Ed.), ISBN: 978-953-307-054-4, InTech, Available from: http://www.intechopen.com/books/carbon-nanotubes/carbon-nanotubes-interconnectsfor-nanoelectronics-circuits

\section{INTECH}

open science | open minds

\author{
InTech Europe \\ University Campus STeP Ri \\ Slavka Krautzeka 83/A \\ 51000 Rijeka, Croatia \\ Phone: +385 (51) 770447 \\ Fax: +385 (51) 686166 \\ www.intechopen.com
}

\author{
InTech China \\ Unit 405, Office Block, Hotel Equatorial Shanghai \\ No.65, Yan An Road (West), Shanghai, 200040, China \\ 中国上海市延安西路65号上海国际贵都大饭店办公楼405单元 \\ Phone: +86-21-62489820 \\ Fax: $+86-21-62489821$
}


(C) 2010 The Author(s). Licensee IntechOpen. This chapter is distributed under the terms of the Creative Commons Attribution-NonCommercialShareAlike-3.0 License, which permits use, distribution and reproduction for non-commercial purposes, provided the original is properly cited and derivative works building on this content are distributed under the same license. 